\title{
A Study on Kohenen Network based on Path Determination for Efficient Moving Trajectory on Mobile Robot
}

\author{
TaeSeok Jin* $^{*}$ and HanHo Tack ${ }^{* * *}$ \\ ${ }^{*}$ Dept. of Mechatronics Engineering, DongSeo University \\ *** Dept. of Electronics Engineering, Jinju National University \\ Tel : +82-51-320-1541, Fax : +82-51-320-1751, E-mail : jints@dongseo.ac.kr
}

\begin{abstract}
We propose an approach to estimate the real-time moving trajectory of an object in this paper. The object's position is obtained from the image data of a CCD camera, while a state estimator predicts the linear and angular velocities of the moving object. To overcome the uncertainties and noises residing in the input data, a Extended Kalman Filter(EKF) and neural networks are utilized cooperatively. Since the EKF needs to approximate a nonlinear system into a linear model in order to estimate the states, there still exist errors as well as uncertainties. To resolve this problem, in this approach the Kohonen networks, which have a high adaptability to the memory of the inputoutput relationship, are utilized for the nonlinear region. In addition to this, the Kohonen network, as a sort of neural network, can effectively adapt to the dynamic variations and become robust against noises. This approach is derived from the observation that the Kohonen network is a type of self-organized map and is spatially oriented, which makes it suitable for determining the trajectories of moving objects. The superiority of the proposed algorithm compared with the EKF is demonstrated through real experiments.
\end{abstract}

Key Words : Distributed cameras, Object tracking, Color histogram, Global model, Intelligent environment.

\section{Introduction}

Detection of moving objects has been utilized in industrial robotic systems, for example, in the recognition and monitoring of unmanned systems that also require compression of moving images [1-2]. Trajectory prediction of moving objects is required for a mobile manipulator that aims at the control and observation of motion information such as object position, velocity, and acceleration. Prediction and estimation algorithms have generally been required for industrial robots. For a simple example, in a pick-and-place operation with a manipulator, the precise motion estimation of the object on the conveyor belt is a critical factor in stable grasping. A well-structured environment, such as the moving-jig that carries the object on the conveyor belt and stops when the manipulator grasps the object, might obviate the motion estimation requirement. However, a well-structured environment limits the flexibility of the production system, requires skillful designers for the jig, and incurs a high maintenance expense; eventually it will disappear from automated production lines. To overcome these problems, a camera needs to detect a moving object stably without stopping the motion, the trajectory prediction of the moving object on the conveyor belt is necessary. The manipulator control system needs to estimate the most accurate position, velocity, and acceleration atany instance to capture the moving object safely without collision and to pick up the object stably without slippage. When the motion trajectory is

Manuscript received Nov. 30, 2009; revised Jun. 11, 2010;

Accepted Jun. 12, 2010.

** Corresponding author not highly random and continuous, it can be modeled analytically to predict the near-future values based on previously measured data [2].

In this paper, a novel approach for the real-time trajectory estimation of a moving object is proposed. For image-data capturing, a CCD camera was utilized. Through a geometrical analysis of the camera and the object, the position of the object could be estimated [3]. There are several approaches in which the state estimator is used to predict the linear and angular velocities of a moving object. The most general approach known so far is the Kalman filter, the performance of which is well verified by numerous studies [4-8]. However, the Kalman filter is not properly applicable to the unstructured environment, even though the adaptive or extended Kalman filter has been proposed to improve prediction accuracy [9]. To make the system robust against noises in the input data and uncertainties, in this approach, artificial neural networks are incorporated into the Kalman filter. Since the artificial neural networks are trained by only the relationship between the input and output, this approach is expected to have higher flexibility than the adaptive or extended Kalman filter. Among the several advantages of the neural networks, the adaptability to variations is well matched to this problem. That is, it has robust characteristics against measurement noises. Fig. 1 summarizes the trajectory estimation system for this research. The input for the dynamic model comes from either the Kalman filter or SOM(Self-Organized Map) according to the following decision equation:

$$
\text { preicted value }=k \cdot \text { Kalman }_{\text {out }}+(1-k) \cdot S O M_{\text {out }}
$$


where $\mathrm{k}=1$ for error $\leq$ threshold and $\mathrm{k}=0$ for error $>$ threshold. The threshold value is empirically determined based on the size of the estimated position error.

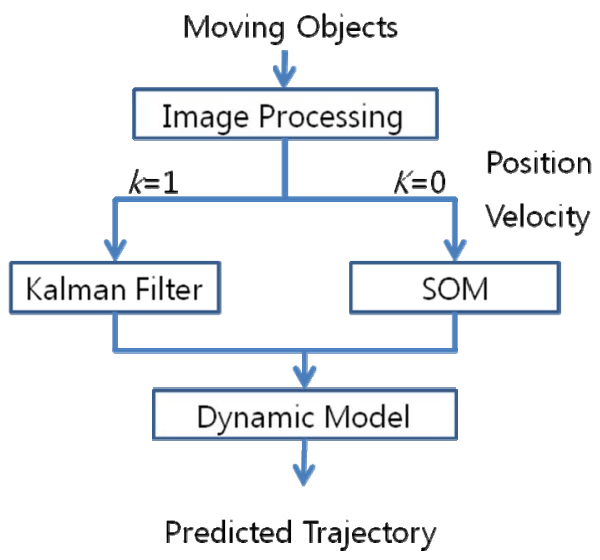

Fig. 1 Trajectory estimation system

\section{Parameter Learning}

\subsection{Pre-Processing}

Classifying the moving-object pattern in the dynamically changing unstructured environment has not yet been tackled successfully [13]. Therefore, in this research, the camera was fixed on a stable platform in order to capture static environment images. To estimate the states of the motion characteristics, the trajectory of the moving object was pre-recorded and analyzed. Figures 2(a), (b), (c) and (d) represent the object images at (t-3), $(\mathrm{t}-2),(\mathrm{t}-1)$ and $(\mathrm{t})$ instance, respectively.

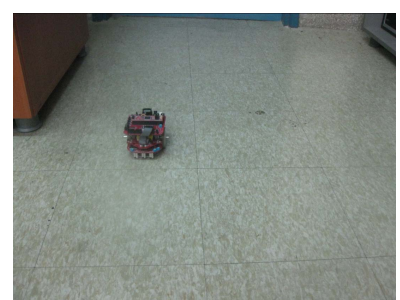

(a)

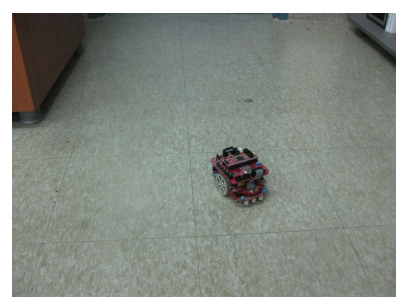

(c)

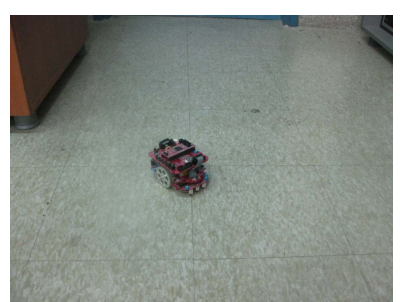

(b)

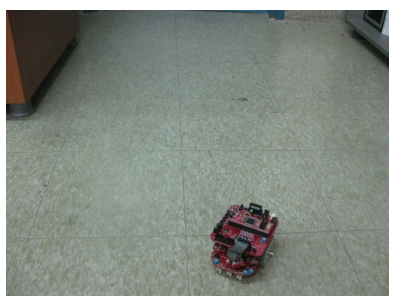

(d)
Fig. 2 Image at the each instance: (a) (d) Image at (t-3), (t-2), $(t-1),(t)$ instance, respectively.

As recognized in the images, most parts of the CCD image correspond to the background. After eliminating the background, the difference between the two consecutive image frames can be obtained to estimate the moving-object motion. To compute the difference, either the absolute values of the two image frames or the assigned values can be used. The difference method is popular in image pre-processing for extracting desired information from the whole image frame [14], which can be expressed as

$$
\operatorname{Output}(x, y)=\operatorname{Image}(x, y)+\operatorname{Image} 2(x, y)
$$

The difference image between Figs. 2(a) and (b) is represented in Fig. 3. When the difference image for the whole time interval can be obtained, the trajectory of the moving object can be calculated precisely.

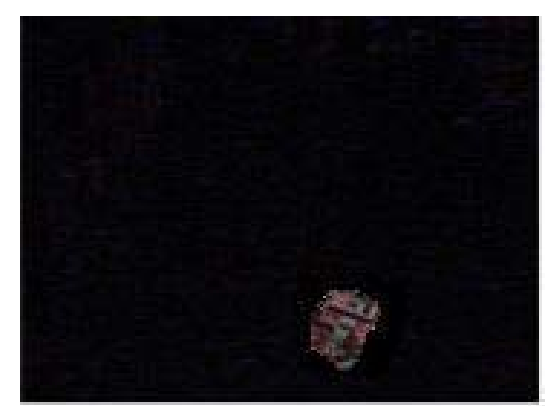

Fig. 3 Difference image between $(t)$ and ( $(-1)$ instance images

\subsection{Motion vector estimation}

Motion vectors can be defined for the moving object at any instance. In order to predict the motion trajectory using the Kalman filter and SOM, these motion vectors are the essential inputs that must be obtained from the difference images. As mentioned previously, since the CCD camera is fixed on a stable platform, the motion vector can be computed based on the data of the difference vector between the two consecutive image frames and the sampling period. When the SOM is utilized for the estimation of the motion vector, highly correlated motion vectors reside close to each other, which will be explained in Section 4. The fact that motion vectors of neighboring states have a high spatial correlation is utilized for estimating the current motion vector in the motion vector estimation process. An example of a motion vector is illustrated in Fig. 4.

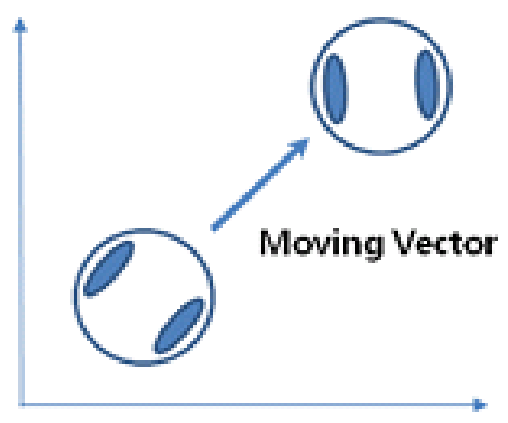

Fig. 4 Illustration of a motion vector 


\section{State Estimation by Kalman Filter}

The input-image data include uncertainties and noises that occur during pre-processing. Therefore, the Kalman filter is suitable for the observer that estimates the states under a noisy environment, since the state transition matrix itself has irregular components [4-5]. Note that the Kalman filter is a recursive process to minimize the estimation error by multiplying a suitable filter gain by the error between estimated and measured values. In the problem of state estimation of a moving object, the measurement vector for the Kalman filter is derived from the position of the moving object on the xy-plane, which position is obtained from the image frames. Using the measurement vector, the state variables, (x, y) position, direction, and linear/angular velocities, are estimated.

In obtaining the filter gain, a zero-mean variance matrix for the estimation error is included, which can be estimated by the terms of the zero-mean variance matrix, state transition matrix, and zero-mean measurement noise matrix, $Q_{k-1}$, of the previous instance, as

$$
\mathbf{P}_{k}^{\prime}=\mathbf{A}_{k, k-1} \mathbf{P}_{k-1} \mathbf{A}_{k, k-1}^{T}+\mathbf{Q}_{k-1}
$$

Now the optimal filter gain, $\mathbf{K}_{k}$, to minimize the state estimation error can be obtained as

$$
\mathbf{K}_{k}=\mathbf{P}_{k}^{\prime} \mathbf{C}_{k}^{T}\left[\mathbf{C}_{k} \mathbf{P}_{k}^{\prime} \mathbf{C}_{k}^{T}+\mathbf{R}_{k}\right]^{-1}
$$

where $\mathbf{P}_{k}^{\prime}$ is the zero-mean variance matrix for the estimation error, $\mathbf{C}_{k}$ is the observation matrix, and $\mathbf{R}_{k}$ represents the zero-mean variance matrix for the measurement noises.

The states are estimated by the following state transition equation, in which an innovation term is added as an input and multiplied by the Kalman filter gain, $\mathrm{K}_{k}$, which is the difference between the measurement vector, $\mathbf{y}_{k}$, and the estimated output using the data from the previous step:

$$
\hat{x}_{k}=\mathbf{A}_{k, k-1} \hat{x}_{k-1}+\mathbf{K}_{k}\left[\mathbf{y}_{k}-\mathbf{C}_{k} \mathbf{A}_{k, k-1} \hat{x}_{k-1}\right]
$$

where $\mathbf{C}_{k} \mathbf{A}_{k, k-1} \hat{x}_{k-1}$ represents the estimated output, $\hat{y}_{k}$.

Before going back to Eq. (5) for the next step, the zero-mean variance matrix of the estimated error needs to be modified as [6-7].

$$
\mathbf{P}_{k}=\mathbf{P}_{k}^{\prime}-\mathbf{K}_{k} \mathbf{C}_{k} \mathbf{P}_{k}^{\prime}
$$

\section{Self-Organized Map}

For a nonlinear system, the Kalman filter requires a process to approximate to a quasi-linear model to derive the filtering equations, which approximation leads to many estimation errors. For the linear model derivation, the Taylor series expansion is usually adopted to select the number of terms or to select the order of computational complexity that is inversely proportional to the modeling accuracy. Therefore, it suffers from the trade-off between accuracy and complexity in obtaining a linear model and in estimating the state variable, $x_{k}$.

Especially since Kalman filtering is based on the first-order approximation, it neither estimates the states properly all of the time nor guarantees the convergence of the states. The adaptive or extended Kalman filter proposed in order to overcome this difficulty, but again, the adaptive Kalman filter suffers from a too-high computational complexity for real-time control. To avoid all of these difficult calculations, there are several ideas for estimating the states using artificial neural networks [10-12]. In these approaches, the supervised learning schemes that require data on the input and oexity for real-time control. To avoid all of these difficult calculations, there are several ideas for estimating the states using artificial neural networks [10-12]. In these approaches, the supervised learning schemes that require data on the input and oexity for real-time control. To avoid all of these difficult calculations, Each neuron in SOM calculates and maintains the Euclidian distance that represents the closeness of the connection intensity vector and the input vector. Each neuron, which belongs to a group that is formed by the winner through competition, can have only the output. This winner neuron and neighboring neurons are allowed to learn from a specific input vector. The connection intensity between the winner neuron $j$ and the neighboring neurons is adaptively changed by

$$
w_{i j}(t+1)=w_{i j}(t)+\alpha\left(x_{i}(t)-w_{i j}(t)\right)
$$

where the parameter, $\alpha$, is pre-determined for SOM.

In this paper, SOM estimated the position and velocity of the moving object. During the learning process, SOM determined the optimal states based on the various measurable states. This enables the use of SOM instead of the Kalman filter for the high nonlinear region where noise distributions and uncertainties are poorly modeled statistically.

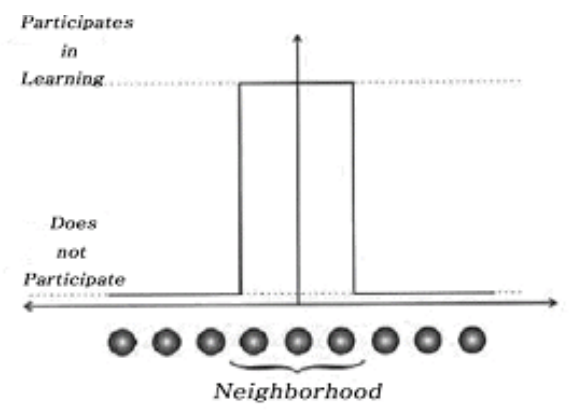

Fig. 5 Competition procedure in SOM

Before the main experiments, the Kohonen networks[12,13] were trained for the moving object, an autonomous micromouse designed for this research. The pattern classifications of learning results for the velocity and orientation, respectively, are illustrated in Fig. 5. 
The velocity and orientation of the moving object can be grouped into several regions, and inside each region, a specific neuron can be selected by real-time learning. The hybrid neural networks formed by the velocity and orientation networks are implemented to achieve more efficient learning and to clearly show that the motion characteristics of the moving object can be classified into several patterns. Since Kohonen networks have learning abilities as neural networks, the un-experienced input coming from the estimation process can be classified into one of the groups, and inside the group region it can be learned for a specific state value. For supervised learning, the input patterns are artificially provided, by humans a priori, and the neural networks learn only the direction to the object patterns from the given input patterns. However, un-supervised learning such as SOM can determine the intrinsic features from the arbitrary input patterns, and can compete with different neurons for the different features. SOM can be efficiently utilized for the trajectory estimation of a moving object when the nonlinearity of the moving object is too high to be estimated by the Kalman filter. Limiting the application of SOM to the nonlinear region is proposed in order to save learning patterns. Many precise learning patterns are required for learning both the linear and nonlinear regions. An insufficient number of learning patterns might lead to erroneous learning results [13]. Since SOM utilizes the dynamic model for trajectory estimation described in Eq. (7), the stochastic characteristics are not essential for SOM, but are definitely required for the Kalman filter.

\section{Experiments}

In the present study, a micro-mouse was designed for a moving object using the microprocessor, ATmega126, which generates a non-programmed trace with the maximum speed of $15 \mathrm{Cm} / \mathrm{sec}$. To show the effectiveness of the SOM, three experiments were performed to show the following as: 1. A relatively linear motion can be estimated properly by the Kalman filter, 2. The extended Kalman filter is somewhat effective for reducing the estimation error in the nonlinear region, and 3. The SOM improves the estimation performance significantly compared with the extended Kalman filter.

\subsection{Comparison between the EKF and SOM}

Before comparing the extended Kalman filter with SOM, some experiments were performed to confirm the superiority of the unsupervised learning method over the supervised learning method [11-12], and the results are summarized in Table 1.

As shown in Table 1, even though supervised learning also has outstanding performance, the performance degradation becomes severe and becomes unsuitable for the dynamically changing environment, whereas unsupervised learning remains consistent. Based on this observation, SOM is selected as the best alternative to the extended Kalman filter for the nonlinear region. To reduce the estimation error, the extended Kalman filter and SOM [10-11] was applied for the nonlinear region, and the results are shown in Fig. 6. The estimation error for the nonlinear region is reduced by $50 \%$ by the extended Kalman filter. However, the maximum error is still larger than $1 \mathrm{Cm}$. Another experiment was performed with SOM instead of the extended Kalman filter in the nonlinear region to show the superiority of the unsupervised learning scheme, SOM. Figure 6 shows the experimental results of state estimation by the Kalman filter for the linear region and by SOM for the nonlinear region (from $\mathrm{S}$ to $\mathrm{T}$ ). We need to focus on the nonlinear region where SOM is applied for the estimation instead of the extended Kalman filter. By comparison of Fig. $6(\mathrm{a})$ and $6(\mathrm{~b})$, it is recognized that SOM is much better than the extended Kalman filter for estimating the nonlinear region. The maximum estimation error by the $\mathrm{SOM}$ is $0.7 \mathrm{Cm}$, which is about $70 \%$ of that by the extended Kalman filter.

Table 1. Performance comparison of two different neural networks

\begin{tabular}{|c|c|c|}
\hline $\begin{array}{c}\text { Classification } \\
\text { Class }\end{array}$ & Number of trials & Number of success* \\
\hline $\begin{array}{c}\text { Supervised } \\
\text { Learning }\end{array}$ & 50 & 39 \\
\hline $\begin{array}{c}\text { Unsupervised } \\
\text { Learning }\end{array}$ & 50 & 46 \\
\hline
\end{tabular}

* A success is tallied when the estimation error is less than 1 $\mathrm{Cm}$.

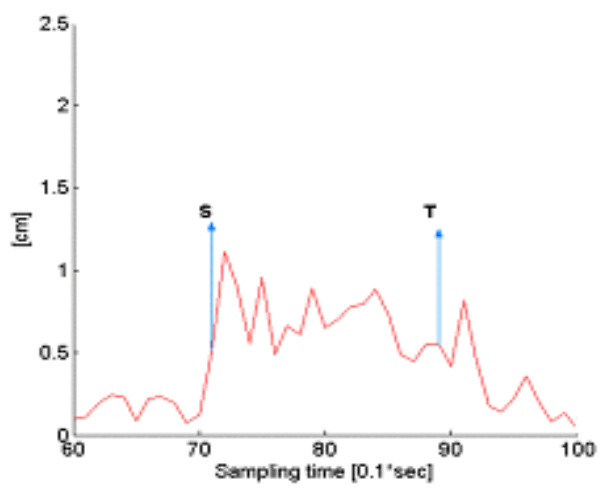

(a) EKF

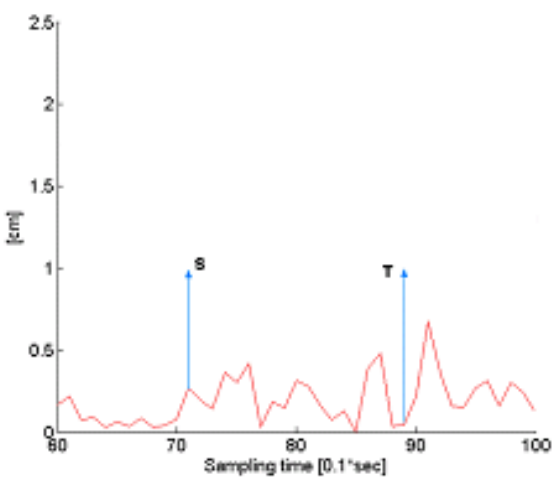

(b) $\mathrm{SOM}$

Fig. 6 Position estimation error in the nonlinear region 


\subsection{Tracking Experiment}

To compare the tracking performance of a mobile robot using the algorithms of the extended Kalman filter, and SOM, experiments of capturing a micro mouse with random motion by the mobile robot were performed. As can be recognized from Fig. 7, SOM provided better performance to the mobile robot in capturing the random motion object than the other algorithms. The mobile robot with SOM has a smooth curve to capture the moving object. As the result, the capturing time for the moving object is the shortest with SOM, as is illustrated in Fig. 7(b). And Figure 8 shows the mobile robot trajectory, the moving object trajectory with each of the algorithms, which comparison concludes that SOM provides a smooth and stable trajectory to the mobile robot to capture a random motion object.

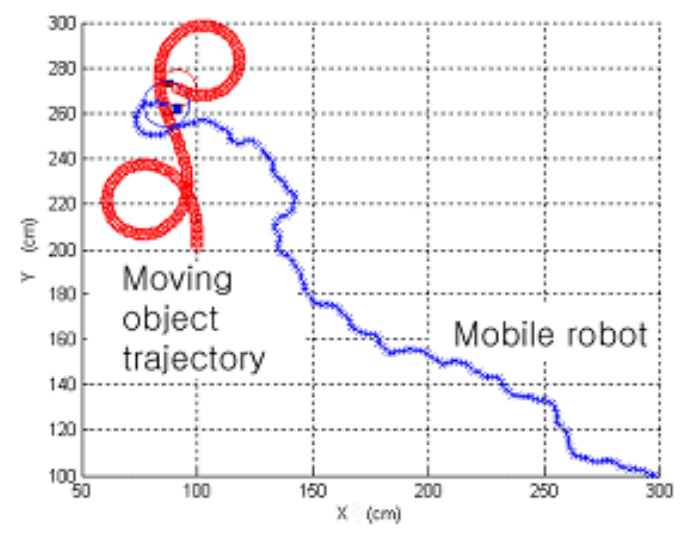

(a) Extended Kalman

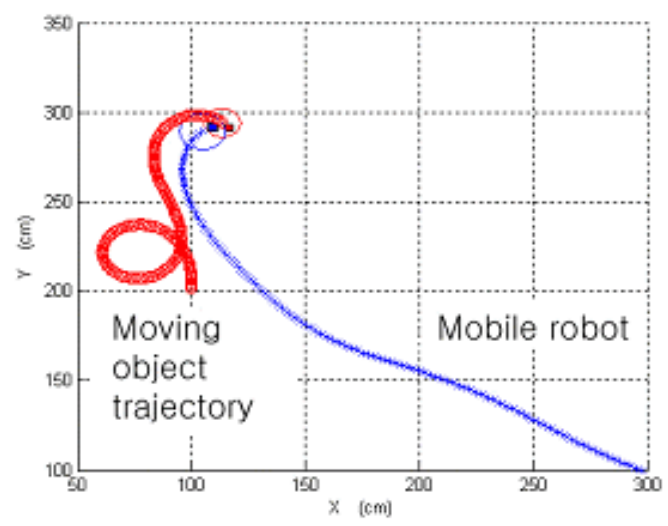

(b) SOM

Fig. 7 Tracking trajectory of moving object and mobile robot using Extended Kalman filter and SOM

\section{Conclusions}

This research proposes a trajectory estimation scheme for a moving object using images captured by a CCD camera. In the scheme, the state estimator has two algorithms: the Kalman filter that estimates the states for the linear approximated region, and SOM for the nonlinear region. The decision for the switchover is made based on the size of the position estimation error that becomes low enough for the linear region and becomes large enough for the nonlinear region. The effectiveness and superiority of the proposed algorithm was verified through experimental data and comparison. The adaptability of the algorithm was also observed during the experiments. For the sake of simplicity, this research was limited to the environment of a fixed-camera view. However, this can be expanded to the moving camera environment, where the input data might suffer from higher noises and uncertainties. As future research, selection of a precise learning pattern for SOM in order to improve the estimation accuracy and the recognition ratio, and development of an illumination robust image processing algorithm, remain.

\section{References}

[1] Kim, Wan C. Hwang, Cheol H. Choi, Su H. Lee, Jang M. "Efficient Tracking of a Moving Object Using Optimal Representative Blocks," ICCAS 2002, International Conference on Control, Automation and Systems, pp. 264-269, 2002.

[2] Shi, K. L. Chan, T. F. Wong, Y. K. Ho, S. L. "Speed Estimation of an Induction Motor Drive Using an Optimized Extended Kalman Filter," IEEE Transactions On Industrial Electronics, vol. 49, no. 1, pp. 124-133, 2002.

[3] Park, J. W. Park, J. H. Hur, H. R. Lee, J. M. Kiyoharu Tagawa, and Hiromassa H. "Capturing a Moving Object using an Active Camera mounted on a Mobile Robot," Proc. of the 5th Int'l Symp. on Artificial Life and Robotics, pp. 609-612, 2000.

[4] Allen, P. K. Tmcenko, A. Yoshimi, B. Michelman, P. "Trajectory filtering and prediction for automated tracking and grasping of a moving object," IEEE International Conference on Robotics and Automation, pp. 1850-1856, 1992.

[5] Ma, Y. Kosecka, J. Sastry, S.S. "Vision guided navigation for a nonholonomic mobile robot," IEEE Transaction on Robotics and Automation, vol. 15, no. 3, pp. 521-536, 1999.

[6] Kalman, R. E. "A new approach to linear filtering and prediction problems," Trans., ASME, J. Basic Eng, Series 82D, pp. 35-45, 1960.

[7] Sorenson, H. W.: Kalman filtering techniques. Advances in Control Systems Theory and Applications, vol.3, pp. 219-292, 1966.

[8] Ramachandra, K. V. "A Kalman tracking filter for estimating position, velocity and acceleration from noisy measurements of a 3-D radar," Electro. Technology, vol. 33, pp. 66-76, 1989.

[9] Chen, Y.Y. Young, K. Y. "An intelligent radar predictor for high-speed moving-target tracking," TENCON '02. Proceedings. IEEE Region 10 Conference on Computers, Communications, Control and Power Engineering, vol. 3, pp. 1638-1641, 2002.

[10]Roberts, J. M. Mills, D. J. Charnley, D. Harris, C. J. "Improved 
Kalman filter initialization using neuro-fuzzy estimation," Int'l. Conf. on Artificial Neural Networks, pp. 329-334, 1995.

[11] DeCruyenaere, J. P. Hafez, H. M. "A comparison between Kalman filters and recurrent neural networks. Neural Networks," IJCNN., Int'l Joint Conference, vol. 4 , pp. 247-251, 1992.

[12] Sorensen, O. "Neural networks for non-linear control. Control Applications," Proceedings of the Third IEEE Conference on, pp. 161-166, 1994.

[13] Partsinevelos, P. Stefanidis, A. Agouris, P. "Automated spatiotemporal scaling for video generalization," Proc. of Int'l. Conference on Image Processing, vol.1, pp.177-180, 2001.

[14] Gonzalez, Rafael G. Woods, Richard E. Digital Image Processing. Addison-Wesley, pp. 47-51, 1993.

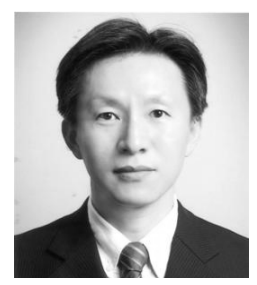

\section{Tae-Seok Jin}

He received the Ph.D. degrees from Pusan National University, Busan, Korea, in 2003, in electronics engineering.

$\mathrm{He}$ is currently an Assistant Professor at DongSeo University. From 2004 to 2005, he was a Postdoctoral Researcher at the Institute of Industrial Science, The University of Tokyo, Japan. His research interests include network sensors fusion, mobile robots, computer vision, and intelligent control. Dr. Jin is a Member of the KIIS, ICROS, and RSJ.

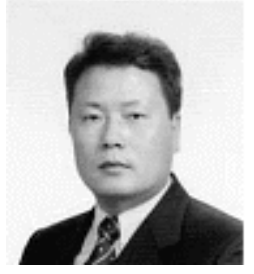

Han-Ho Tack

$\mathrm{He}$ received the B.S. degree in Department of Electronic Engineering from Bukyung National University, Busan Korea, in 1987. He received the M..S. degree in Department of Electronic Engineering from Dong-A University, Busan, Korea, in 1992. He received $\mathrm{Ph}$. D. degree in Department of Electronic \& Communication Engineering from the Korea Maritime University, Busan, Korea, in 1998. From 1987 to 1989 , he was a Researcher at the Laboratory of Hung Chang Co. Ltd. Since 1991, he has been a faculty member of the Electronic Engineering at the Jinju National University, where he is currently a Professor. His research interests are Neural Network, Fuzzy System, Robotics, Factory Automation, Mechanical Vibration, Transfortation, and Multimedia System etc. He is a member of IEEE, KIMISC, KMS, KIEE, and KFIS. 